\title{
MULTIPHASE DESIGN OF AUTONOMIC SELF-HEALING THERMOPLASTIC ELASTOMERS
}

\author{
Y. Chen ${ }^{1}$, A. M. Kushner ${ }^{1}$, G. A. Williams ${ }^{1}$, J. Hentschel ${ }^{1}$ and Z. Guan ${ }^{1}$ \\ ${ }^{1}$ Department of Chemistry, 1102 Natural Sciences 2, University of California, Irvine, CA \\ 92697, USA - e-mail: zguan@uci.edu
}

Keywords: Self-healing, thermoplastic elastomer, multiphase material, supramolecular, brush copolymer

\section{ABSTRACT}

The development of polymers that can spontaneously repair themselves after mechanical damage would significantly improve the safety, lifetime, energy efficiency, and environmental impact of manmade materials. Most approaches to self-healing materials either require the input of external energy, or need healing agents, solvent or plasticizer. Despite intense research in this area, the synthesis of a stiff material with intrinsic self-healing ability remains a key challenge. Our laboratory has recently succeeded in a design of multiphase supramolecular thermoplastic elastomers that combine high modulus and toughness with spontaneous healing capability. In one design, H-bonding brush polymers (HBPs) self-assemble into hard-soft microphaseseparated system, combining the enhanced stiffness and toughness of hybrid polymers with the self-healing capacity of dynamic supramolecular assemblies [1]. In another design, supramolecular ABA triblock copolymers formed by dimerization of 2ureido-4-pyromidone (UPy) end-functionalized polystyrene-b-poly(n-butylacrylate) (PS-b-PBA) AB diblock copolymers are synthesized, resulting in a self-healing material that combines the advantageous mechanical properties of thermoplastic elastomers and the dynamic self-healing features of supramolecular materials [2]. In contrast to previous self-healing polymers, our systems spontaneously self-heals as a single-component solid material at ambient conditions without the need of any external stimulus, healing agent, plasticizer, or solvent.

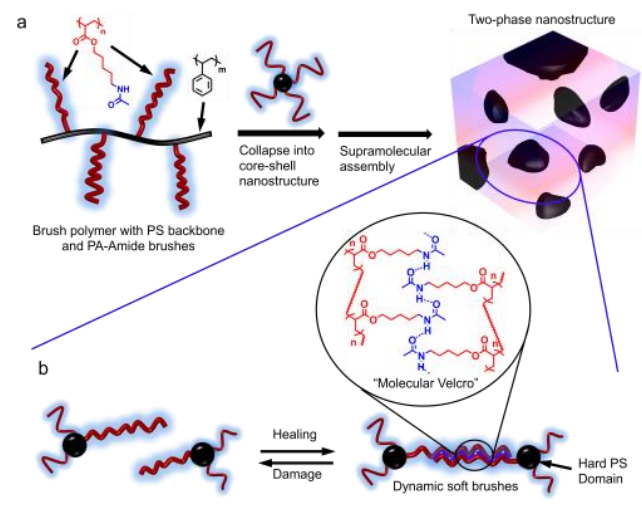

\section{REFERENCES}

[1] Y. Chen, A.M. Kushner, G.A. Williams, Z. Guan, Multiphase design of autonomic self-healing thermoplastic elastomers, Nature Chemistry 4 (2012) 467-472.

[2] J. Hentschel, A.M. Kushner, J. Ziller, Z. Guan, Self-Healing Supramolecular Block Copolymers, Angewandte Chemie International Edition 51 (2012) 10561-10565. 